\title{
Fourier spectrum and shape evolution of an internal Riemann wave of moderate amplitude
}

\author{
E. Kartashova ${ }^{1}$, E. Pelinovsky ${ }^{1,2,3,4}$, and T. Talipova ${ }^{2,3}$ \\ ${ }^{1}$ Institute for Analysis, Johannes Kepler University, Linz, Austria \\ ${ }^{2}$ Department of Nonlinear Geophysical Processes, Institute of Applied Physics, Nizhny Novgorod, Russia \\ ${ }^{3}$ Department of Applied Mathematics, Nizhny Novgorod State Technical University, Nizhny Novgorod, Russia \\ ${ }^{4}$ Department of Information Systems, National Research University - Higher School of Economics, Nizhny Novgorod, Russia
}

Correspondence to: E. Pelinovsky (pelinovsky@ hydro.appl.sci-nnov.ru)

Received: 21 April 2013 - Revised: 2 June 2013 - Accepted: 27 June 2013 - Published: 7 August 2013

\begin{abstract}
The nonlinear deformation of long internal waves in the ocean is studied using the dispersionless Gardner equation. The process of nonlinear wave deformation is determined by the signs of the coefficients of the quadratic and cubic nonlinear terms; the breaking time depends only on their absolute values. The explicit formula for the Fourier spectrum of the deformed Riemann wave is derived and used to investigate the evolution of the spectrum of the initially pure sine wave. It is shown that the spectrum has exponential form for small times and a power asymptotic before breaking. The power asymptotic is universal for arbitrarily chosen coefficients of the nonlinear terms and has a slope close to $-8 / 3$.
\end{abstract}

\section{Introduction}

Internal waves in the ocean are an important object of nonlinear geophysical hydrodynamics, discussed in numerous books and reviews (e.g., Ostrovsky and Stepanyants, 1989; Baines, 1995; Grimshaw et al., 1998, 2007; Lyapidevsky and Teshukov, 2000; Miropolsky, 2002; Staquet and Sommeria, 2002; Grimshaw, 2002, 2007; Vlasenko et al., 2005; Helfrich and Melville, 2006; and Hutter, 2012). The breaking of internal waves contributes significantly to the mixing processes in the ocean and has been frequently observed in real nature (Smyth and Holloway, 1988; Henyey and Hoering, 1997; Afanasyev and Pelter, 2001; Chant and Wilson, 2000; Horn et al., 2001; Konyaev and Filonov, 2006).
Strongly nonlinear deformation and breaking of internal waves is described theoretically in the framework of twolayer flows with no dispersion (Baines, 1995; Klemp et al., 1997; Lyapidevsky and Teshukov, 2000; Jiang and Smith, 2001; Holland et al., 2002; Fringer and Street, 2003; Milewski et al., 2004; Sakai and Redekopp, 2007; Zahibo et al., 2007). In fact, the long interfacial waves in a twolayer fluid as described in the Boussinesq approximation with rigid-lid approximation are described by almost the same equations of motion as surface shallow-water waves, allowing to introduce the Riemann invariants and use the classic theory of nonlinear hyperbolic equations. In comparison with surface shallow-water waves the nonlinear dynamics of interfacial Riemann (sometimes also called simple) waves are significantly richer. In particular, breaking can appear on the front-slope or back-slope of the wave depending on the thicknesses of layers of uniform density in the medium (Zahibo et al., 2007).

The possibility of Riemann waves to occur in a more complicated stratified fluid containing several layers or with continuous stratification is discussed by Chumakova et al. (2009). A solution has been found for a smoothly stratified basin (Ostrovsky and Helfrich, 2011), where it is shown numerically that a simple wave can emerge from a wide class of initial disturbances. It is important to mention that they find strongly nonlinear Riemann waves not only for the first mode, but also for the second and third modes. Their evolution leads to wave breaking and the formation of hydraulic lumps or bore-like pulses with different decay rates depending on the modal number. This nonlinear process influences the modal and spectral distribution of the internal 
wave field (Yermakov and Pelinovsky, 1975; Filonov and Novotryasov, 2007; Novotryasov and Karnaukhov, 2009).

In the present paper we study analytically long nonlinearly deformed internal waves of moderate amplitude in the ocean with arbitrary but stable stratification in density and shear flow. Our mathematical model is based on the dispersionless Gardner (extended Korteweg-de Vries) equation briefly discussed in Sect. 2. The shape deformation of internal waves resulting from different values and signs of the coefficients of the quadratic and cubic nonlinear terms is analyzed in Sect. 3. Spectra of Riemann waves are discussed in Sect. 4. In particular it is demonstrated that before breaking, the spectrum has a power asymptotic for all possible values of nonlinear terms. The results obtained are summarized in the Conclusions (Sect. 5).

\section{Dispersionless Gardner equation for long internal waves}

The Korteweg-de Vries equation and its generalizations are a popular model to describe weakly nonlinear and weakly dispersive internal waves (Ostrovsky and Stepanyants, 1989; Grimshaw et al., 1998, 2007; Miropolsky, 2002; Grimshaw, 2002, 2007; Vlasenko et al., 2005; Helfrich and Melville, 2006; Hutter, 2012). In many cases of the vertical distribution of density and shear flow the coefficient of the quadratic nonlinear term is small (for instance, it is zero for a two-layer flow with equal thicknesses), and the cubic nonlinear term should be accounted in the same order of perturbation theory as the quadratic nonlinear term. This combined Kortewegde Vries equation is called the Gardner equation, and it is very often used to model the solitary internal waves in the ocean with real stratification (Grimshaw et al., 2007, 2010a), or to demonstrate that modulation instability leads to the formation of rogue internal waves (Grimshaw et al., 2010b; Talipova et al., 2011), or to describe transformation of the internal tide in the coastal zone (Holloway et al., 1999). In the latter case in the initial stage of the transformation dispersion effects are negligible (Smyth and Holloway, 1988; Sakai and Redekopp, 2007; Zahibo et al., 2007) and so the dispersionless Gardner equation can be chosen as an appropriate model:

$$
\frac{\partial \eta}{\partial t}+V(\eta) \frac{\partial \eta}{\partial x}=0, \quad V(\eta)=\alpha \eta+\beta \eta^{2},
$$

where $\eta$ is vertical displacement of isopycne with maximal oscillations. Here the coefficients of the quadratic and cubic nonlinearity are

$$
\alpha=\left(\frac{3}{2}\right) \frac{\int_{0}^{H}(c-U)^{2}(\mathrm{~d} \Phi / \mathrm{d} z)^{3} \mathrm{~d} z}{\int_{0}^{H}(c-U)(\mathrm{d} \Phi / \mathrm{d} z)^{2} \mathrm{~d} z},
$$

$$
\begin{aligned}
& \beta=\frac{3}{2} \\
& \frac{\int \mathrm{d} z\left\{(c-U)^{2}\left[3(\mathrm{~d} T / \mathrm{d} z)-2(\mathrm{~d} \Phi / \mathrm{d} z)^{2}\right](\mathrm{d} \Phi / \mathrm{d} z)^{2}-\alpha^{2}(\mathrm{~d} \Phi / \mathrm{d} z)^{2}+\Pi\right\}}{\int(c-U)(\mathrm{d} \Phi / \mathrm{d} z)^{2} \mathrm{~d} z}, \\
& \Pi=\alpha(c-U)\left[5(\mathrm{~d} \Phi / \mathrm{d} z)^{2}-4 \mathrm{~d} T / \mathrm{d} z\right] \mathrm{d} \Phi / \mathrm{d} z,
\end{aligned}
$$

and $\Phi(z)$ is the eigenfunction determined by the eigenvalue problem:

$$
\frac{\mathrm{d}}{\mathrm{d} z}\left[(c-U(z))^{2} \frac{\mathrm{d} \Phi}{\mathrm{d} z}\right]+N^{2}(z) \Phi=0,
$$

with zero boundary conditions on the sea bottom $(z=0)$ and sea surface $(z=H)$, and normalization: $\max [\Phi(z)]=1$. This equation with zero boundary conditions has countable number of eigenmodes, each with its own eigenvalue. As the eigenvalue $\mathrm{c}$ is the long-wave speed of internal waves of any mode, it is not important for our further study which specific mode is analyzed.

The function $T(z)$ is the nonlinear correction to the modal function $\Phi(z)$, which is a solution of the inhomogeneous eigenvalue problem:

$$
\begin{aligned}
& \frac{\mathrm{d}}{\mathrm{d} z}\left[(c-U)^{2} \frac{\mathrm{d} T}{\mathrm{~d} z}\right]+N^{2} T=-\alpha \frac{\mathrm{d}}{\mathrm{d} z}\left[(c-U) \frac{\mathrm{d} \Phi}{\mathrm{d} z}\right] \\
& +\frac{3}{2} \frac{\mathrm{d}}{\mathrm{d} z}\left[(c-U)^{2}\left(\frac{\mathrm{d} \Phi}{\mathrm{d} z}\right)^{2}\right],
\end{aligned}
$$

solved with zero boundary conditions on $z=0$ and $z=H$, and additional condition $T=0$ on the level where the modal function is maximal. Here $N(z)$ and $U(z)$ are the buoyancy frequency and shear flow respectively, and Boussinesq and rigid-lid approximations are used.

The dispersionless Gardner equation describes the long nonlinear internal waves of weak and moderate amplitudes. It may be obtained as a result of the asymptotic procedure. In the case of two-layer stratification (for interfacial waves) it can be obtained from the full, strongly nonlinear Riemann wave equation (Sakai and Redekopp, 2007; Zahibo et al., 2007) or from the dispersionless limit of the OstrovskyGrue equation (Ostrovsky and Grue, 2003) or the ChoiCamassa system (Choi and Camassa, 1999). In the last case it is necessary to use the Taylor expansion for function $V(\eta)$ describing the local velocity of points on the wave surface.

It is important to notice that the coefficients of the nonlinear terms in Eq. (1) may be positive, negative or equal to zero. Moreover, for a very specific case of so-called "symmetric" stratification, both coefficients vanish (Talipova et al., 1999; Kurkina et al., 2011) and the next nonlinear terms in the asymptotic expansion are required; this case is not analyzed below.

In the present study we assume that both nonlinear terms do not vanish simultaneously, but any one of them might have a zero coefficient. 
If both coefficients are not zero, Eq. (1) can be reduced to canonical dimensionless form:

$$
\frac{\partial u}{\partial t}+V(u) \frac{\partial u}{\partial x}=0, \quad V(u)=6 u(1 \pm u),
$$

by a change of variables

$u=\frac{\eta}{6}\left|\frac{\beta}{\alpha}\right|, \quad t^{\prime}=\alpha t\left|\frac{\alpha}{\beta}\right|$.

Below, both forms - dimensioned and dimensionless - of the dispersionless Gardner equation will be used to analyze the process of nonlinear wave deformation and the evolution of its Fourier spectrum.

\section{Nonlinear wave deformation}

Here we begin with the dimensioned form of dispersionless Gardner equation written above as Eq. (1); it can be solved exactly, and its solution is the Riemann wave:

$\eta(x, t)=F[x-V(\eta) t]$,

where $F(x)$ describes the initial wave profile. This solution describes the monotonically deformed wave and is "onevalued" up to the time of breaking, $T$. This can be seen easily regarding the expression for local wave steepness derived from Eq. (9):

$$
\frac{\partial \eta}{\partial x}=\frac{\mathrm{d} F / \mathrm{d} x}{1+\frac{\mathrm{d} V}{\mathrm{~d} x} t} .
$$

Where the initial slope $\mathrm{d} V / \mathrm{d} x$ is negative, the denominator in Eq. (10) tends to zero with growing $t$, and the local wave steepness becomes infinite. The first time of wave breaking is

$$
T=\frac{1}{(-\mathrm{d} V / \mathrm{d} x)_{\max }} .
$$

Detailed calculation will be done below for a wave of initially sinusoidal form:

$F(x)=A \sin (k x)$,

with arbitrary amplitude $A$ and wave number $k$. Formally, Eq. (12) describes a sin of real arbitrary argument $-\infty<$ $k x<\infty$; however, the analysis below is also valid for one period of $F(x)$, that is, for the argument range $(0-2 \pi)$.

\subsection{Breaking point location}

The first step is determining the location of the breaking point. Differentiation of $V$ with respect to $x$ leads to

$$
\frac{\mathrm{d} V}{\mathrm{~d} x}=\alpha k A[\cos (k x)+\mathrm{CQ} \sin (2 k x)], \quad \mathrm{CQ}=\frac{\beta A}{\alpha} .
$$

Parameter CQ is dimensionless and has evident physical sense as the ratio of cubic to quadratic nonlinearity. The analysis below shows the importance of this parameter: it allows the description of the place of breaking point for the systems with pure quadratic, pure cubic and mixed nonlinearity in the same mathematical frame. Indeed, if $\mathrm{CQ}=0$, then there is no cubic nonlinear term, $\beta=0$; if $\mathrm{CQ}=\infty$, then there is no quadratic nonlinear term, $\alpha=0$. Otherwise parameter CQ can be positive or negative. Without loss of generality it is assumed below that $\alpha \geq 0$.

The wave steepness given by Eq. (13) has negative extremes at

$$
\frac{F *}{A}=\sin (k x *)=-\frac{1}{8 \mathrm{CQ}} \pm \sqrt{\frac{1}{2}+\frac{1}{64 \mathrm{CQ}^{2}}}, \quad \cos (k x *)<0 .
$$

The first root of this equation is

$$
\frac{F_{1}}{A}=\sin \left(k x_{1}\right)=\sqrt{\frac{1}{2}+\frac{1}{64 \mathrm{CQ}^{2}}}-\frac{1}{8 \mathrm{CQ}} .
$$

It exists for any positive value of CQ and varies from $k x_{1}=\pi$ for $\mathrm{CQ}=0$ (pure quadratic nonlinearity) to $k x_{1}=3 \pi / 4$ for $\mathrm{CQ}=\infty$ (pure cubic nonlinearity). The breaking point on the wave profile moves from $F=0$ for $\mathrm{CQ}=0$ to $F=A(2)^{-1 / 2}$ for $\mathrm{CQ}=\infty$.

The second root,

$\frac{F_{2}}{A}=\sin \left(k x_{2}\right)=-\sqrt{\frac{1}{2}+\frac{1}{64 \mathrm{CQ}^{2}}}-\frac{1}{8 \mathrm{CQ}}$,

exists only if $\mathrm{CQ}>1 / 2$ (cubic nonlinear term prevails on quadratic one). The breaking point is initially at $k x_{2}=3 \pi / 2$ (on the wave trough) and moves to $7 \pi / 4$ with increase in CQ.

In Fig. 1a it is shown how the locations of the breaking points depend on the parameter CQ; the initial sine wave is regarded for the case when both cubic and quadratic coefficients are positive: $\alpha>0$ and $\beta>0$. The case, when cubic and quadratic terms have different signs, $\alpha>0$ and $\beta<0$ is shown in Fig. $1 \mathrm{~b}$.

\subsection{Breaking time}

In the previous section we have computed the maximal values of the derivative $\mathrm{d} V / \mathrm{d} x$. However, to apply the formula for the breaking time, Eq. (11), we also need to compare the values of it in the roots, Eqs. (15) and (16). Notice that even if both roots exist (in case $|C Q|>1 / 2$ ) they behave differently, because the slopes $\mathrm{d} V / \mathrm{d} x$ differ in both points and $|\mathrm{d} V / \mathrm{d} x|$ has absolute maximum on the first root. This means that at first instance wave breaking will be near phase $k x=\pi$ and later in the second point. Only in the case of pure cubic nonlinearity wave breaking will occur at both points simultaneously. 


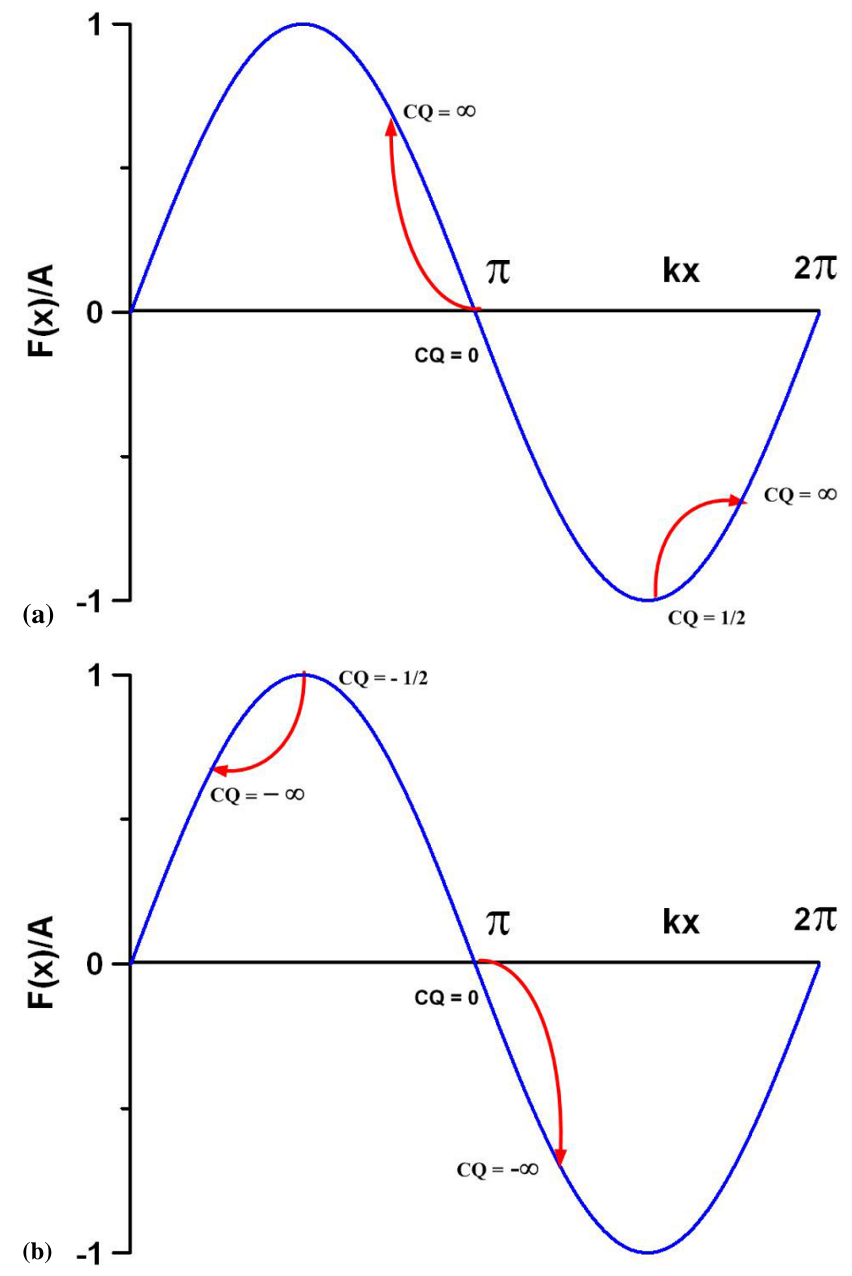

Fig. 1. Motion of bifurcation points with change in CQ: (a) $\beta>0$, and (b) $\beta<0$.

In the case of purely quadratic nonlinearity $(\beta=0)$, the breaking time is

$T_{2}=\frac{1}{|\alpha| k A}$.

In the case of purely cubic nonlinearity $(\alpha=0)$, the breaking time is

$T_{3}=\frac{1}{|\beta| k A^{2}}$.

The influence of the cubic nonlinear term on the breaking time computed from Eq. (11) is shown in Fig. 2. As one may expect, positive cubic nonlinearity decreases the breaking time. Negative cubic nonlinearity decreases the absolute value of local nonlinear speed, which also decreases the breaking time. Even more, the total breaking time is a function of $|\mathrm{CQ}|$ only.

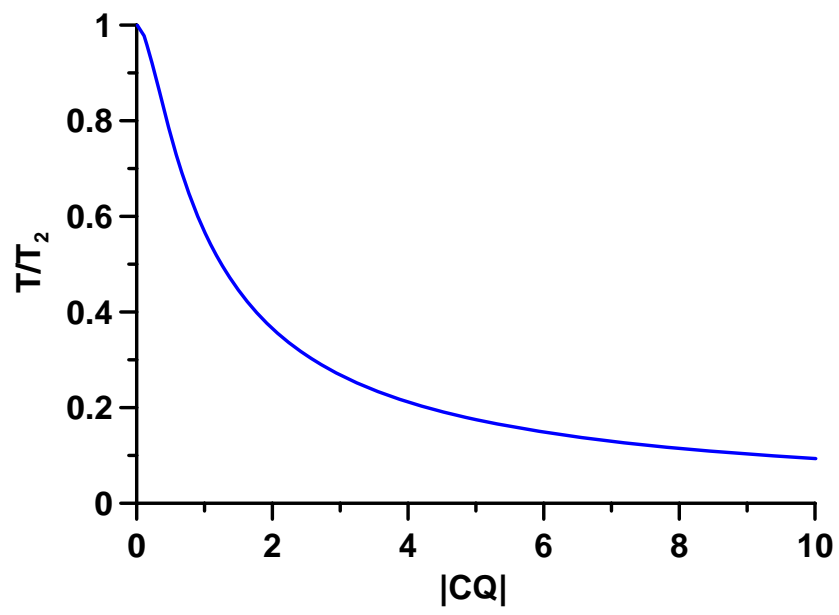

Fig. 2. Breaking time versus $C Q\left(T_{2}\right.$ is the breaking time in a purely quadratic medium).

\subsection{Time evolution of the wave shape}

Nonlinear deformation of the initially sinusoidal wave, Eq. (12), can be investigated in detail using the exact solution, Eq. (9). Figure 3 demonstrates the deformation in the limiting cases: (a) $\alpha>0, \beta=0(\mathrm{CQ}=0)$; (b) $\alpha=$ $0, \beta>0(\mathrm{CQ}=+\infty)$ at different stages of development (time is normalized on breaking time, which is $T_{2}$ for pure quadratic medium and $T_{3}$ for pure cubic nonlinear medium). Only one shock is formed in the case of purely quadratic nonlinearity, and two shocks simultaneously in the case of purely cubic nonlinearity. In case of mixed nonlinearity we will use "canonical" values for the coefficients: $\alpha=6$, and $\beta= \pm 6$, which amounts to $\mathrm{CQ}= \pm A$.

Below we consider the general case, when coefficients of the dispersionless Gardner equation have canonical values and $\mathrm{CQ}=+A$. Figure 4 a displays the wave deformation for $A=0.4$ at times $0.3,0.4$ (breaking time), and 0.5 ; there exists only one breaking point. Of course, "multi-valued" solutions of the Eq. (1) after breaking time shown in Fig. 4 do not materialize in real physical media and are given here only for illustration of the breaking process to show the asymmetry with respect to the horizontal axis.

With increase in amplitude $(A=2)$, the cubic nonlinear term becomes important $(\mathrm{CQ}=2)$ and we see the formation of two shock fronts, faster on the crest and slower on the trough (Fig. 4b).

Figure 5 demonstrates the different character of the nonlinear wave deformation for positive and negative sign of the cubic nonlinearity. In the latter case breaking occurs on back slope of the wave shape.

Qualitatively, the same character of nonlinear deformation is obtained for strongly nonlinear interfacial waves (Sakai and Redekopp, 2007; Zahibo et al., 2007), where the cubic nonlinear term is always negative. 


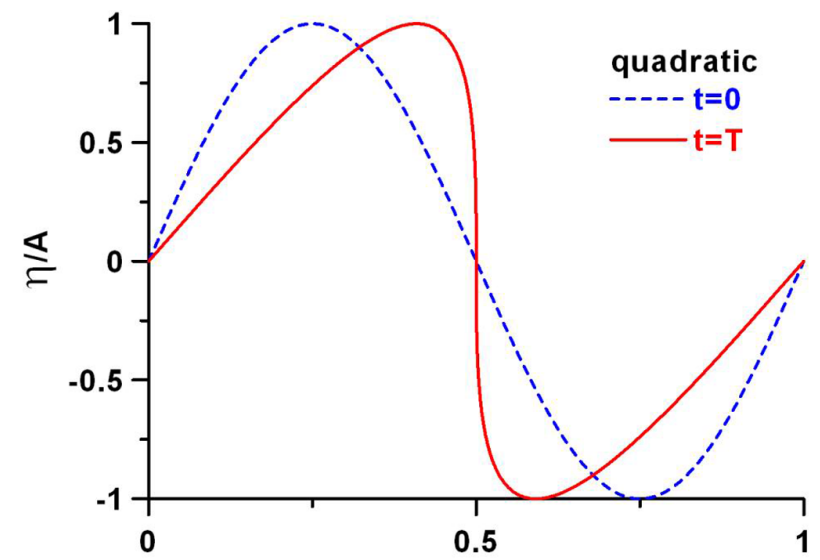

(a)

$k x / 2 \pi$

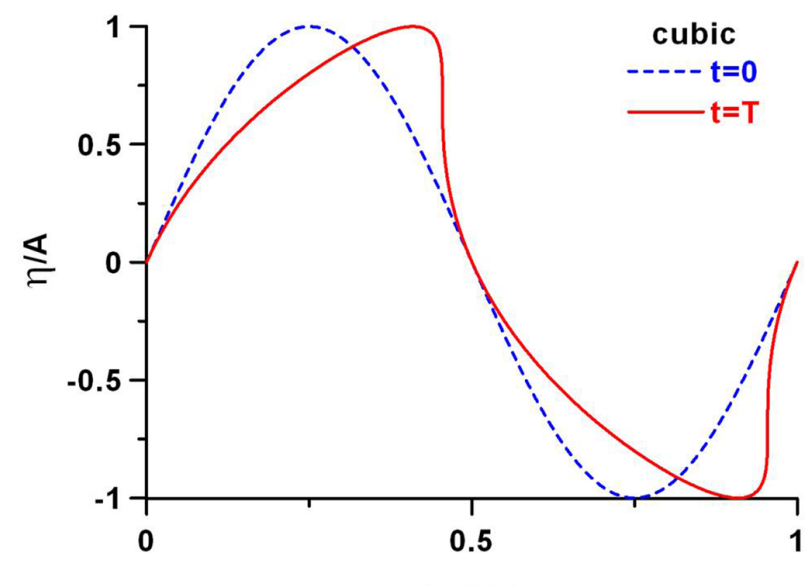

(b)

$\mathrm{kx} / 2 \pi$

Fig. 3. Nonlinear wave transformation: (a) $\alpha>0, \beta=0(\mathrm{CQ}=0)$; (b) $\alpha=0, \beta>0(\mathrm{CQ}=+\infty)$. Breaking time $T$ is $T_{2}$ for (a) and $T_{3}$ for (b).

\section{Fourier spectrum of a nonlinearly deformed wave}

It is known that a periodic wave can be represented by the Fourier series:

$\eta(x, t)=\frac{a_{0}(t)}{2}+\sum_{n=1}^{\infty}\left[a_{n}(t) \cos (n k x)+b_{n}(t) \sin (n k x)\right]$,

where the coefficients of Fourier series are defined as

$S_{n}(t)=a_{n}+i b_{n}=\frac{k}{\pi} \int_{0}^{2 \pi / k} \eta(x, t) \exp [i n k x] \mathrm{d} x$.

The problem to calculate integrals in Eq. (20) is that the Riemann wave, Eq. (9), is given inexplicitly. Here we show what can be done in this case.

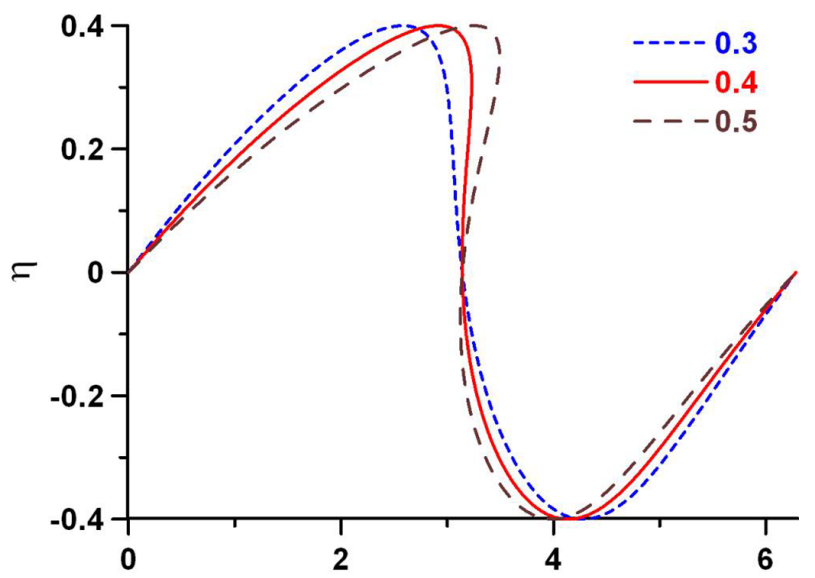

(a)

$\mathbf{k X}$

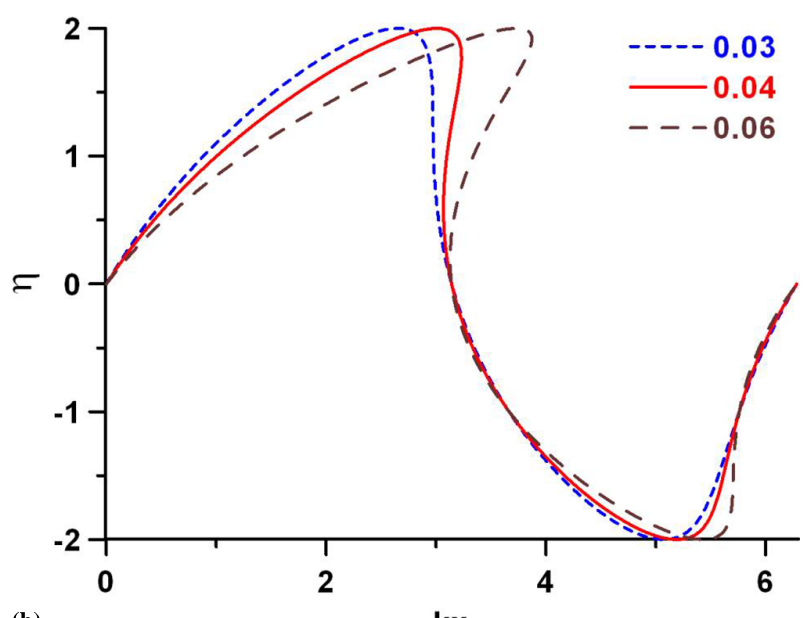

(b)

Fig. 4. Nonlinear wave deformation in the case of positive sign of the cubic nonlinearity. (a): $\mathrm{CQ}=A=0.4$, and (b) $\mathrm{CQ}=A=2$. Numbers - different times.

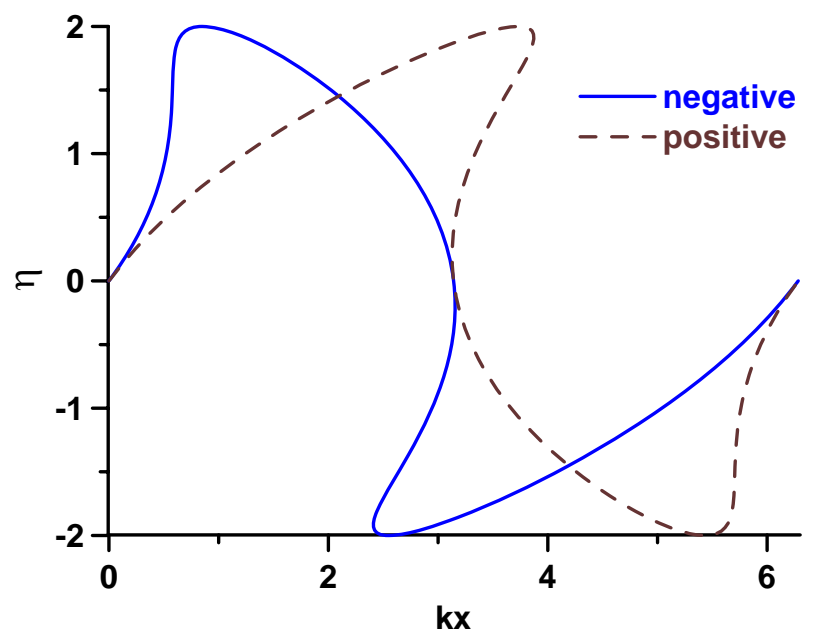

Fig. 5. Nonlinear wave deformation for different signs of cubic nonlinearity. $A=2, t=0.06$. 
It is convenient to perform the change of variables in Eq. (20):

$y=x-V(\eta) t$,

which replaces $\mathrm{d} x$ by

$\mathrm{d} x=\frac{\mathrm{d} y}{1-t \frac{\mathrm{d} V}{\mathrm{~d} \eta} \frac{\partial \eta}{\partial x}}$.

Taking into account expression (10), formula (22) can be rewritten as

$\mathrm{d} x=\left[1+t \frac{\mathrm{d} V}{\mathrm{~d} F} \frac{\mathrm{d} F}{\mathrm{~d} y}\right] \mathrm{d} y$.

Accordingly, the spectrum $S_{n}$ takes the form:

$$
\begin{aligned}
& S_{n}(t)=\frac{k}{\pi} \int_{0}^{2 \pi / k} F(y)\left[1+t \frac{\mathrm{d} V}{\mathrm{~d} F} \frac{\mathrm{d} F}{\mathrm{~d} y}\right] \\
& \exp \{\operatorname{ink}[y+V(F) t]\} \mathrm{d} y,
\end{aligned}
$$

where $F(y)=A \sin (k y)$ is the initial sine wave. Simple manipulation in Eq. (24) reduces it to

$S_{n}(t)=\frac{i}{n \pi} \int_{0}^{2 \pi / k} \frac{\mathrm{d} U}{\mathrm{~d} y} \exp \{i n k[y+V(U) t]\} \mathrm{d} y$.

Substitution of $x=k y$ yields the final expression for the wave spectrum of the following form:

$$
\begin{aligned}
& S_{n}(t)=\frac{i A}{n \pi} \int_{0}^{2 \pi} \cos x \\
& \quad \exp \left\{i n\left[x+\left(\alpha k A \sin x+\beta k A^{2} \sin ^{2} x\right) t\right]\right\} \mathrm{d} x .
\end{aligned}
$$

Introducing new variables:

$p(t)=\alpha k A t, \quad q(t)=\beta k t A^{2} / 2$,

we can rewrite Eq. (26) in a more convenient form:

$S_{n}=\frac{i A}{n \pi} \exp (i n q) \int_{0}^{2 \pi} \cos x \exp [p n \sin x] \exp [-q n \cos (2 x)]$

$\exp [i n x] \mathrm{d} x$.

This allows using the formulas for Bessel functions (formulas 8.511-4 in Gradshteyn and Ryzhik, 2007):

$\exp [i z \cos \phi]=\sum_{m=-\infty}^{m=+\infty} i^{m} J_{m}(z) \exp [i m \phi]$,
$\exp [i z \sin \phi]=\sum_{m=-\infty}^{m=+\infty}(-1)^{m} J_{m}(z) \exp [-i m \phi]$, to rewrite the integral on the RHS (right-hand side) of Eq. (28) as a double sum:

$$
\begin{aligned}
& S_{n}=\frac{i A}{n \pi} \exp (i n q) \sum_{m=-\infty}^{m=+\infty} \sum_{l=-\infty}^{l=+\infty}(-1)^{m} i^{l} J_{m}(n p) J_{l}(-n q) \\
& \int_{0}^{2 \pi} \cos x \exp [i(n+2 l-m) x] \mathrm{d} x .
\end{aligned}
$$

Now the integral on the RHS of Eq. (30) can be computed as

$$
\begin{aligned}
& \int_{0}^{2 \pi} \cos x \exp [i(n+2 l-m) x] \mathrm{d} x \\
& =\pi\left[\delta_{n+2 l-m+1}+\delta_{n+2 l-m-1}\right],
\end{aligned}
$$

where $\delta_{j}$ is the Kronecker symbol, which is equal to 1 if its index is zero, and zero for other indexes, that is $\delta_{0}=1$ and $\delta_{j}=0$ if $j \neq 0$.

So the double sum in Eq. (30) can be transformed into a single sum as

$$
\begin{aligned}
& S_{n}=(-1)^{n-1} \frac{2 i A}{n^{2} p} \exp (i n q) \sum_{l=-\infty}^{l=+\infty}(-i)^{l}(n+2 l) \\
& J_{n+2 l}(n p) J_{l}(n q),
\end{aligned}
$$

where the following formulas for Bessel functions (Gradshteyn and Ryzhik, 2007) are used:

$J_{n+1}(z)+J_{n-1}(z)=\frac{2 n}{z} J_{n}(z), J_{n}(-z)=(-1)^{n} J_{n}(z)$.

In the case of pure quadratic nonlinearity $(\beta=0)$, expression (32) is simplified to

$S_{n}=(-1)^{n-1} \frac{2 i A}{n p} J_{n}(n p)$,

and coincides with the famous Bessel-Fubini spectrum of a Riemann wave in nonlinear acoustics (Pelinovskii, 1976; Rudenko and Soluyan, 1977). The Riemann wave may be represented by a Fourier series containing only sine terms. In Fig. 6 temporal evolution of the energy spectrum $E_{n}=$ $\left|S_{n} / A\right|^{2}$ is shown, for the case of pure quadratic nonlinearity. Using formulas (17) for $T_{2}$ and (27) for $p$, it is easy to show that in Eq. (34) $p=t / T_{2}$. Accordingly, we demonstrate graphically the spectrum evolution in terms of times $1 / 4$, $1 / 2,3 / 4$, and 1 normalized over $T_{2}$. One can clearly see the formation of a power asymptotic with slope of $-8 / 3$. As it is shown in Kartashova and Pelinovsky (2013) this asymptotic is related to the formation of a singularity in the wave shape at breaking time, of the form $x^{1 / 3}$. 


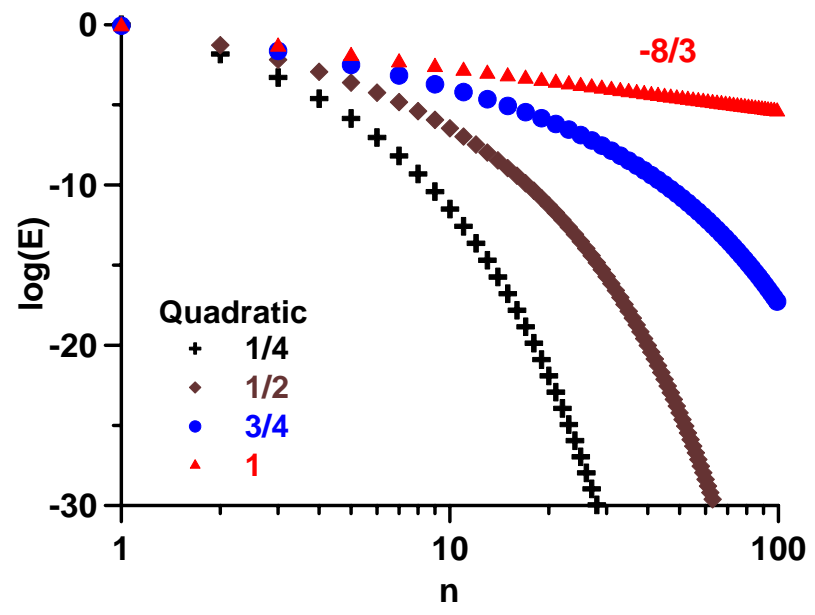

Fig. 6. Energy spectra $\left|S_{n} / A\right|^{2}$ of a Riemann wave in purely quadratic nonlinear medium. Time is normalized on $T_{2}$.

In the case of purely cubic nonlinearity $(\alpha=0)$, expression (32) simplifies to $(n=2 m+1)$,

$$
\begin{aligned}
& S_{m}=\frac{i A}{2 m+1} \exp [i(2 m+1) q]\left\{J_{m}([2 m+1] q)\right. \\
& \left.\quad+i J_{m+1}([2 m+1] q)\right\} .
\end{aligned}
$$

It contains both sine and cosine terms in the Fourier series, and moreover the phase of each harmonic is shifted (Pelinovskii, 1976; Kartashova and Pelinovsky, 2013).

In Fig. 7 temporal evolution of the energy spectrum is shown, for the case of pure cubic nonlinearity. Using formulas (18) for $T_{3}$ and (27) for $q$, it is easy to show that in Eq. (35) $q=t / 2 T_{3}$. Accordingly, we demonstrate graphically the spectrum evolution in terms of times $1 / 4$, $1 / 2,3 / 4$, and 1 normalized over $T_{3}$.

It is interesting to note that the "breaking spectrum asymptotic" in the case of a purely cubic nonlinearity has a slope of $-8 / 3$, the same as in the purely quadratic case discussed above. This fact has been first established in Kartashova and Pelinovsky (2013).

In the general case with $\alpha \neq 0$ and $\beta \neq 0$, it is convenient to rewrite Eq. (32) using the sum on positive integers only.

$$
\begin{aligned}
S_{n} & =(-1)^{n-1} \frac{2 i A}{n^{2} p} \exp (i n q)\left\{n J_{n}(n p) J_{0}(n q)\right. \\
& +\sum_{m=1}^{m=+\infty}(-i)^{m} J_{m}(n q)\left[(n+2 m) J_{n+2 m}(n p)\right. \\
& \left.\left.+(n-2 m) J_{n-2 m}(n p)\right]\right\}
\end{aligned}
$$

For applications (e.g., in geophysics) it is important to have also the energy spectrum of a Riemann wave, which can be

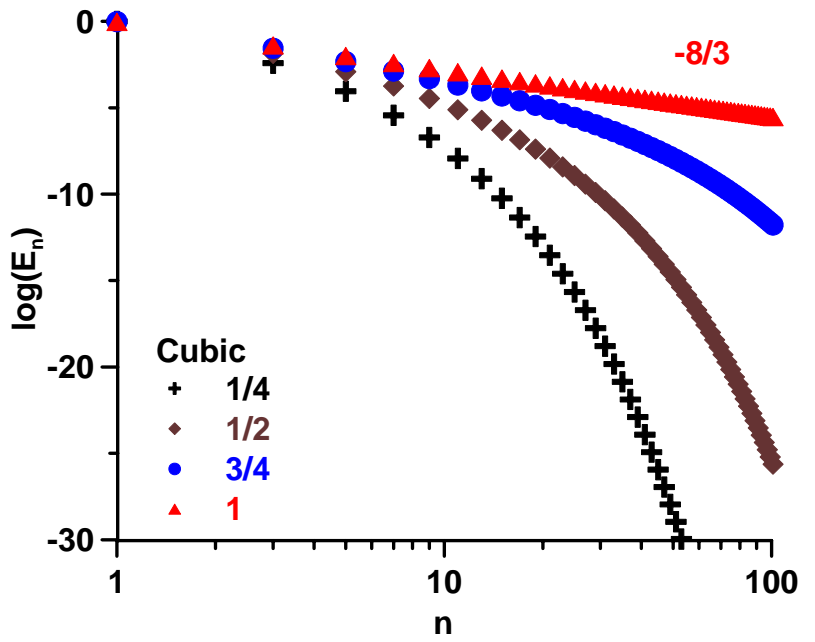

Fig. 7. Energy spectra of a Riemann wave in purely cubic nonlinear medium. Time is normalized on $T_{3}$ accordingly.

computed as

$E_{n}=\left|S_{n} / A\right|^{2}=\frac{4}{n^{4} p^{2}}\left[W_{1 n}^{2}+W_{2 n}^{2}\right]$,

where

$$
\begin{aligned}
& W_{1 n}=n J_{n}(n p) J_{0}(n q)+\sum_{m=1}^{m=+\infty}(-1)^{m} J_{2 m}(n q) \\
& {\left[(n+4 m) J_{n+4 m}(n p)+(n-4 m) J_{n-4 m}(n p)\right]} \\
& W_{2 n}=\sum_{m=0}^{m=+\infty}(-1)^{m} J_{2 m+1}(n q)\left[(n+4 m+2) J_{n+4 m+2}(n p)\right. \\
& \left.+(n-4 m-2) J_{n-4 m-2}(n p)\right] .
\end{aligned}
$$

For the graphical presentations of the general case we use canonic values of the parameters of nonlinearity: $\alpha=6$, and $\beta= \pm 6$. For this choice of values we have $\mathrm{CQ}= \pm A$. Using the formulas (27) for $p$ and $q$ we conclude that $q=$ $\pm A p / 2=\mathrm{CQ} p / 2$. As it is shown above, $p=t / T_{2}$. Figure 8 below demonstrates the difference between the spectrum of a Riemann wave with purely quadratic nonlinearity $(C Q=0$, shown in black) and the spectrum of a Riemann wave with small positive cubic nonlinearity $(\mathrm{CQ}=0.2$, shown in blue) for relatively small times $\left(t / T_{2}=0.5\right)$. For comparison, the breaking spectrum asymptotic $(-8 / 3)$ is also shown, in red. As expected, being far from the breaking point both pure quadratic and cubic-quadratic curves (black and blue correspondingly) are lower than the red asymptotic. Moreover, the cubic-quadratic curve lies higher than the purely quadratic curve as cubic effects lead to an increase of the total energy. 


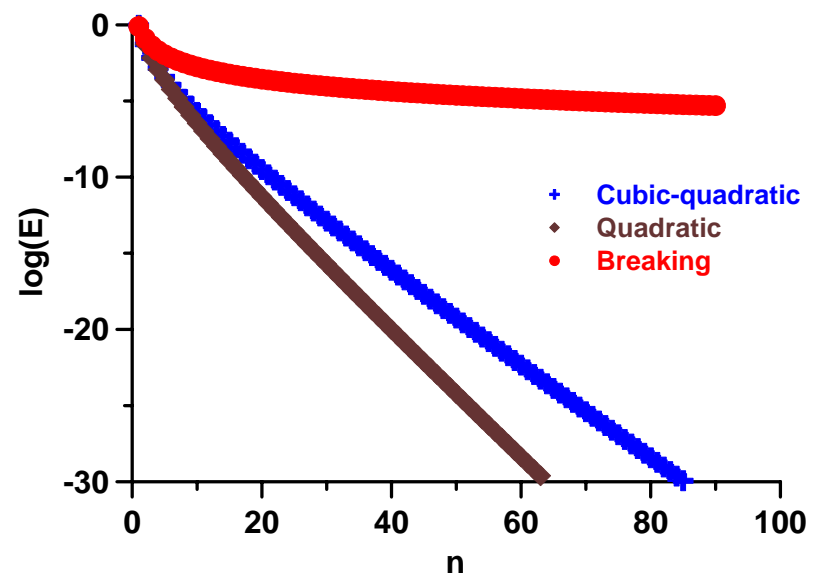

Fig. 8. Spectrum of a Riemann wave, far from breaking point $\left(t / T_{2}=0.5\right)$, for $\mathrm{CQ}=0$ (brown) and $\mathrm{CQ}=0.2$ (blue). The breaking power asymptotic $(-8 / 3)$ is shown in red.

As it can be seen in Fig. 8, the spectrum for both purely quadratic and combined cubic-quadratic nonlinearity has exponential shape. We will demonstrate this analytically for purely quadratic nonlinearity. Using the tangent approximation of Bessel functions (formula 8.452 given in Gradshteyn and Ryzhik, 2007):

$J_{n}(n p) \approx \frac{\exp \left\{n\left[\sqrt{1-p^{2}}-\operatorname{acosh}(1 / p)\right]\right\}}{\sqrt{2 n \pi \sqrt{1-p^{2}}}}$,

the spectrum of Eq. (34) transforms for small times $(p \ll 1)$ and big $n$ to

$S_{n} \approx(-1)^{n-1} \frac{2 i A}{n p \sqrt{2 n \pi}} \exp [-n \ln (2 / p)]$,

and the existence of the exponential (or quasi-exponential) asymptotic is evident.

Figure 9 shows the spectrum of a Riemann wave at breaking time for the same value $\mathrm{CQ}=0.2$ as in the previous example (in blue) together with the breaking power asymptotic $-8 / 3$ (in red) for the limiting cases of purely quadratic and purely cubic nonlinearity. It is clearly seen that the same asymptotic applies for the cubic-quadratic case. This is a very important result showing the existence of the universal asymptotic $-8 / 3$ for any cubic-quadratic nonlinearity.

In Fig. 10 we present the spectrum at breaking time for much bigger cubic nonlinearity $(C Q=1)$. An increase of the cubic nonlinearity leads to inhomogeneous energy transfer for the lowest harmonics (Fig. 10), which can be interpreted in the following way. A quadratic nonlinearity generates all harmonics, that is, odd and even, whereas a cubic nonlinearity generates only odd harmonics. This provides

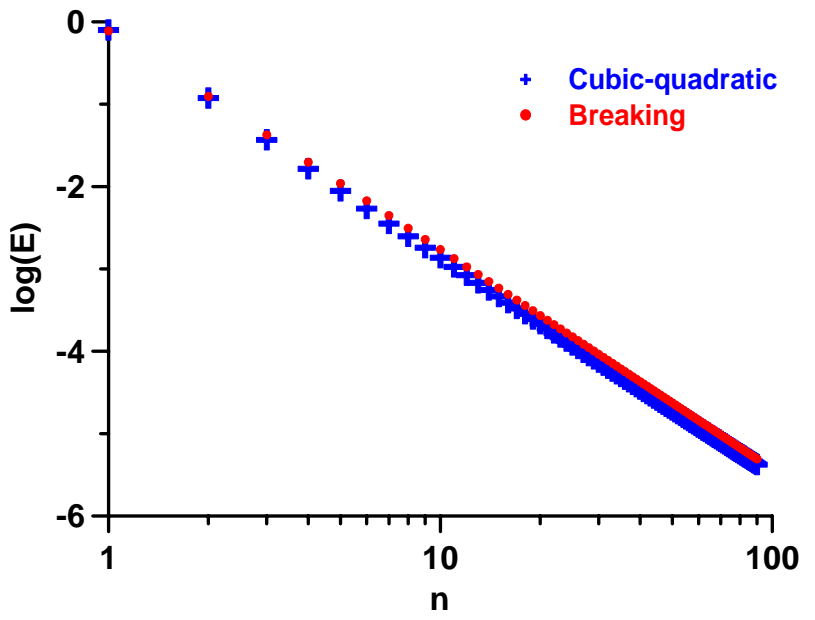

Fig. 9. Spectrum of a Riemann wave for a case $C Q=0.2$ at breaking time.

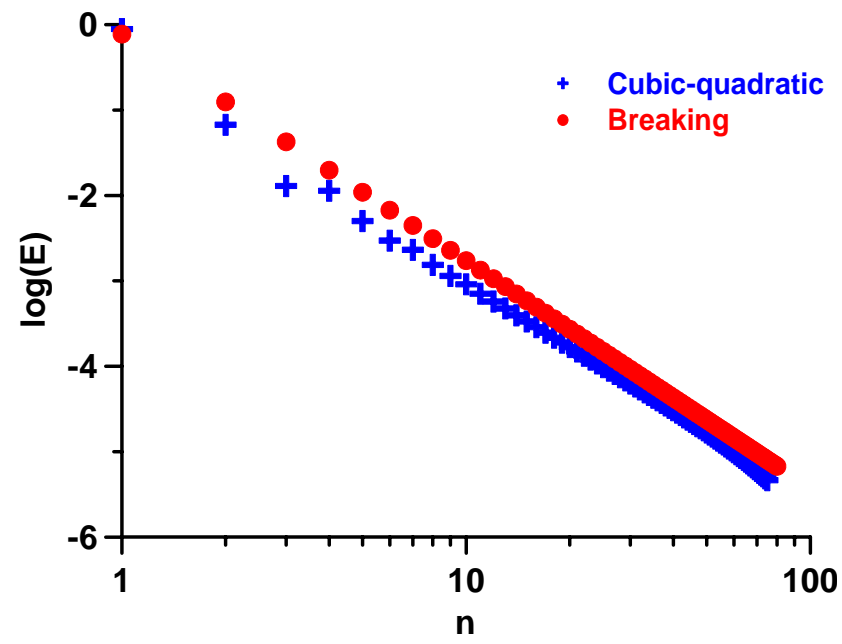

Fig. 10. Spectrum of a Riemann wave with $C Q=1$ at breaking time against breaking power asymptotic.

some explanation that for low harmonics the spectrum follows a zigzag line with higher values for even harmonics. However, this effect disappears for harmonics above 10, and we have again the universal power asymptotic.

In Fig. 11 we show that non-uniformity of the energy transfer increases with bigger nonlinearity $(\mathrm{CQ}=5)$ and is visible in the spectral domain also for higher harmonics (up to 20 ).

Notice that there must be a limit to this effect, because, if CQ goes to infinity, the nonlinearity becomes purely cubic and the spectrum tends to the uniform breaking asymptotic shown in Fig. 7b.

Finally, we note that the energy of a Riemann wave does not depend on the sign of the cubic nonlinear term. This may be seen in Fig. 5: the curves for positive and negative values of parameter $\beta$ can be transformed into each other 


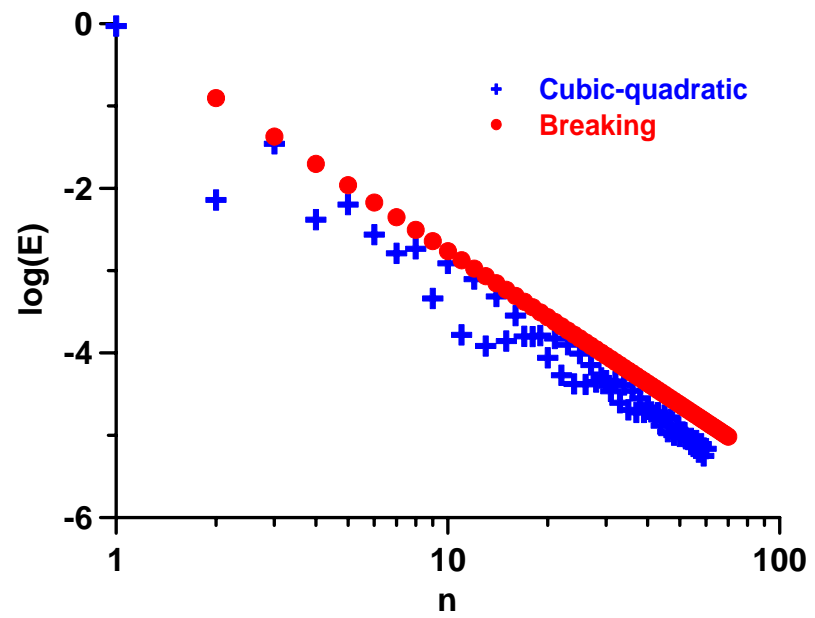

Fig. 11. Spectrum of a breaking Riemann wave for a case of $C Q=$ 5 .

by changing the signs of amplitude and time, which does not change the Fourier spectrum, so the energy of both wave shapes is the same.

\section{Conclusions}

In previous studies the structure of a Riemann internal wave was analyzed for a two-layer flow and for the case of a negative cubic nonlinear term (Smyth and Holloway, 1988; Sakai and Redekopp, 2007; Zahibo et al., 2007).

In this study we use the dispersionless Gardner equation in a more elaborate form to study a Riemann internal wave in a medium with arbitrary stable stratification in density and shear flow and generic nonlinearity, which means that the coefficients of the quadratic and cubic nonlinear terms may have arbitrary signs but do not vanish simultaneously.

The main results are the following:

- The time for breaking to occur depends only on the absolute values of the coefficients of the quadratic and cubic nonlinear terms but not on their signs and it decreases with increasing wave amplitude. The shock appears on the face or back slope depending on the signs and ratio of the quadratic and cubic nonlinear terms.

- Using the dispersionless Gardner equation, the spectrum evolution of an initially sinusoidal wave has been analyzed and an explicit formula for the Fourier spectrum in terms of Bessel functions obtained. The asymptotic behavior of the Fourier spectrum has been studied in detail.

- The spectrum can be described by an exponential law for small times and has a power asymptotic describing the form of the singularity in the wave shape at the point where the wave breaks at the time of breaking.
- The energy spectrum of the Riemann wave at the point of breaking is universal for any kind of nonlinearity and described by a power law with a slope close to $-8 / 3$.

One has to understand clearly that after breaking, the solution given by the Gardner equation for the form of the Riemann wave is not valid anymore and the Gardner equation has to be modified taking into account dispersive and dissipative effects. The study of the evolution of the Fourier spectrum in this case will be done in the future.

Acknowledgements. The authors acknowledge support by the Austrian Science Foundation (FWF) under projects P22943 and P24671. EP acknowledges also VolkswagenStiftung, RFBR grants (11-05-00216, 13-05-97037, 13-02-00656) and Federal Targeted Program "Research and educational personnel of innovation Russia" for 2009-2013. TT acknowledges RFBR grants (12-0500472, 12-05-33070 and 13-05-90424).

Edited by: R. Grimshaw

Reviewed by: Y. A. Stepanyants and one anonymous referee

\section{References}

Afanasyev, Ya. D. and Pelter, W. R.: On breaking internal waves over the sill in Knight Inlet, P. Roy. Soc. Lond. A Mat., 457, 2799-2825, 2001.

Baines, P. G.: Topographic effects in stratified flows, Cambridge University Press, Cambridge, UK, 1995.

Chant, R. and Wilson, R.: Internal hydraulics and mixing in highly stratified estuary, J. Geophys. Res., 105, 14215-14222, 2000.

Choi, W. and Camassa, R.: Fully nonlinear internal waves in a twofluid system, J. Fluid Mech., 396, 1-36, 1999.

Chumakova, L., Menzaque, F. E., Milewski, P. A., Rosales, R. R., Tabak, E. G., and Turner, C. V.: Shear instability for stratified hydrostatic flows, Commun. Pur. Appl. Math., 62, 183-197, 2009.

Filonov, A. and Novotryasov, V.: On a spectrum of nonlinear internal waves in the oceanic coastal zone, Nonlin. Processes Geophys., 14, 757-762, doi:10.5194/npg-14-757-2007, 2007.

Fringer, O. B. and Street, R. L.: The dynamics of breaking progressive interfacial waves, J. Fluid Mech., 494, 319-353, 2003.

Gradshteyn, I. S. and Ryzhik, I. M.: Table of integrals, series, and products, Academic Press, Elsevier, 2007.

Grimshaw, R. (Ed.): Environmental stratified flows, Kluwer Academic Publishers New York, Boston, Dordrecht, London, Moscow, 2002.

Grimshaw, R. (Ed.): Solitary Waves in Fluids, WIT Press, Southampton, Boston, USA, 2007

Grimshaw, R., Pelinovsky, E., and Talipova, T.: Modeling internal solitary waves in the coastal ocean, Surv. Geophys., 28, 273-298, 2007.

Grimshaw, R., Pelinovsky, E., Talipova, T., and Kurkina, O.: Internal solitary waves: propagation, deformation and disintegration, Nonlin. Processes Geophys., 17, 633-649, doi:10.5194/npg-17633-2010, 2010a. 
Grimshaw, R., Pelinovsky, E., Talipova, T., and Sergeeva, A.: Rogue internal waves in the ocean: long wave model, The European Physical Journal Special Topics, 185, 195-208, doi:10.1140/epjst/e2010-01249-4, 2010b.

Grimshaw, R. H. J., Ostrovsky, L. A., Shrira, V. I., and Stepanyants, Y. A.: Long nonlinear surface and internal gravity waves in a rotating ocean, Surv. Geophys., 19, 289-338, 1998.

Helfrich, K. R. and Melville, W. K.: Long nonlinear internal waves, Annu. Rev. Fluid Mech., 38, 395-425, 2006.

Henyey, F. S. and Hoering, A.: Energetic of borelike internal waves, J. Geophys. Res., 102, 3323-3330, doi:10.1029/96JC03558, 1997.

Holland, D., Rosales, R., Stefanica, D., and Tabak, E.: Internal hydraulic jumps and mixing in two-layer flows, J. Fluid Mech., 470, 63-83, 2002.

Holloway, P., Pelinovsky, E., and Talipova, T.: A Generalised Korteweg - de Vries model of internal tide transformation in the coastal zone, J. Geophys. Res., 104, 18333-18350, 1999.

Horn, D. A., Imberger, J., and Ivey, G. N.: The degeneration of large-scale interfacial gravity waves in lakes, J. Fluid Mech., 434, 181-207, 2001.

Hutter, K. (Ed.): Nonlinear internal waves in lakes, in: Advances in Geophysical and Environmental Mechanics and Mathematics, Springer-Verlag Berlin Heidelberg, 2012.

Jiang, Q. and Smith, R.: Ideal shocks in 2-layer flow. Part I: Under a rigid lid, Tellus A, 53, 129-145, doi:10.1034/j.16000870.2001.00096.x, 2001.

Kartashova, E. and Pelinovsky, E.: Universal breaking point asymptotic for energy spectrum of Riemann waves in weakly nonlinear non-dispersive media, Phys. Rev. E, submitted, arXiv:1303.2885, 2013.

Klemp, J. B., Rottuno, R., and Skamarock, W. C.: On the propagation of internal bores, J. Fluid Mech., 331, 81-106, 1997.

Konyaev, K. and Filonov, A.: Breaking of a tidal internal waves on a steep shelf as Inferred from Temperature Measurements, Izv. Atmos. Ocean. Phy., 42, 523-530, 2006.

Kurkina, O. E., Kurkin, A. A., Soomere, T., Pelinovsky, E. N., and Ruvinskaya, E. A.: Higher-order $(2+4)$ Korteweg-de Vries - like equation for interfacial waves in a symmetric three-layer fluid, Phys. Fluids, 23, 116602, doi:10.1063/1.3657816, 2011.

Lyapidevsky, V. Yu. and Teshukov, V. M.: Mathematical models of long wave propagation in inhomogeneous fluid, Nauka, Novosibirsk, 2000.

Milewski, P. A., Tabak, E. G., Turner, C. V., Rosales, R. R., and Menzaque, F.: Nonlinear stability of two-layer flows, Commun. Math. Sci., 2, 427-442, 2004.
Miropolsky, Yu. Z.: Dynamics of internal gravity waves in the ocean, Kluwer, 2001.

Novotryasov, V. V. and Karnaukhov, A. S.: Nonlinear Interaction of Internal Waves in the Coastal Zone of the Sea of Japan, Izv. Atmos. Ocean. Phy., 45, 262-270, 2009.

Ostrovsky, L. A. and Grue, J.: Evolution equations for strongly nonlinear internal waves, Phys. Fluids, 15, 2934-2948, 2003.

Ostrovsky, L. A. and Helfrich, K. R.: Strongly nonlinear, simple internal waves in continuously-stratified, shallow fluids, Nonlin. Processes Geophys., 18, 91-102, doi:10.5194/npg-18-91-2011, 2011.

Ostrovsky, L. A. and Stepanyants, Y. A.: Do internal solitons exist in the ocean?, Rev. Geophys., 27, 293-310, doi:10.1029/RG027i003p00293, 1989.

Pelinovskii, E. N.: Spectral analysis of simple waves, Radiophys. Quantum El., 19, 262-270, 1976.

Rudenko, O. V. and Soluyan, S. I.: Theoretical foundations of nonlinear acoustics, New York, Consultants Bureau, 1977.

Sakai, T. and Redekopp, L. G.: Models for strongly-nonlinear evolution of long internal waves in a two-layer stratification, Nonlin. Processes Geophys., 14, 31-47, doi:10.5194/npg-14-312007, 2007.

Smyth, N. and Holloway, P.: Hydraulic jump and undular bore formation on a shelf break, J. Phys. Oceanogr., 18, 947-962, 1988.

Staquet, C. and Sommeria, J.: Internal gravity waves: from instabilities to turbulence, Annu. Rev. Fluid Mech., 34, 559-593, 2002.

Talipova, T. G., Pelinovsky, E., Lamb, K., Grimshaw, R., and Holloway, P.: Cubic nonlinearity effects in the propagation of intense internal waves, Dokl. Earth Sci., 365, 241-244, 1999.

Talipova, T. G., Pelinovsky, E. N., and Kharif, Ch.: Modulation instability of long internal waves with moderate amplitudes in a stratified horizontally inhomogeneous ocean, JETP Lett., 94, 182-186, 2011.

Vlasenko, V., Stashchuk, N., and Hutter, K.: Baroclinic tides: theoretical modeling and observational evidence, Cambridge University Press, Cambridge, 2005.

Zahibo, N., Slunyaev, A., Talipova, T., Pelinovsky, E., Kurkin, A., and Polukhina, O.: Strongly nonlinear steepening of long interfacial waves, Nonlin. Processes Geophys., 14, 247-256, doi:10.5194/npg-14-247-2007, 2007.

Yermakov, S. A. and Pelinovsky, E. N.: Toward a theory of multimodal distribution of the characteristics of long internal waves of finite amplitude, Izv. Atmos. Ocean. Phys., 11, 10551060, 1975. 